\title{
Mathematical Modeling of Dengue Virus Control and Vaccination
}

\section{Method}

\author{
Anmole Razzaq
}

NCBA \& E

\begin{abstract}
Algebraic modeling the infectious syndrome is expedient to nurture the appliance in pardon method syndrome suckers and in pardon method splendid. Now consume deliberate in ascending order the dengue virus. Now edge the totally limitless Non-Standard Finite Difference (NSFD) construction bound for a mathematical typical the dengue virus. The explain numerical display is limited, dynamically label and comprise the positivity of the explanation, who is central rations so modeling a dominant infectious. The contrast amongst the original NonStandard Finite Change erection, Euler technique and Runge-Kutta organization of instruction four (RK-4) parades the worth of the proposed Non-Standard Finite Change scheme. Explicit NSFD scheme displays meeting the careful evenness truths of the perfect for any value increase or decrease but Euler and RK-4 fail for huge change value.
\end{abstract}

Keywords: Dengue Virus, Dynamical System, Numerical Modeling, Convergence.

DOI: $10.7176 / \mathrm{MTM} / 9-4-01$

Publication date: April $30^{\text {th }} 2019$.

\section{Introduction}

The mathematical modeling for dengue skilled insolence to produce the behavior of condition peoples and the basis, nearly talented hearings of the shown to the stop infection [9-13]. Dynamical models for the spread of ailment objects in a public people, recognized the Kermack and McKendrick SEIR traditional endemic model of suggested [5-8]. Now models bring assessments aimed at consecutive progression of verminous lumps in a people [1-4]. Present day build completely convergent to the algebraic model for the broadcast diminuendos for Dengue who saves the dangerous monies of the relentless model [14-16].

\subsection{Mathematical Model}

\section{A: Variables and Parameters}

$s(t)$ : Susceptible entities class at time $t$.

$\mathrm{e}(\mathrm{t})$ : Exposed individuals class at time $\mathrm{t}$.

$\mathrm{i}(\mathrm{t})$ : Infected individuals class at time $\mathrm{t}$.

$\mathrm{r}(\mathrm{t})$ : Recover individuals class at time $\mathrm{t}$.

$\mu_{h}$ : Rates per-capita mortality human.

$\beta_{h}$ : Force of infection human susceptible.

$\gamma_{h}$ : Period of virus rate in human.

$\sigma_{h}$ : Recover period rate in human.

$M_{1}(t)$ : Ovipositional Rate of treatment. 
$M(t): \quad$ Ovipositional induced mortality rate.
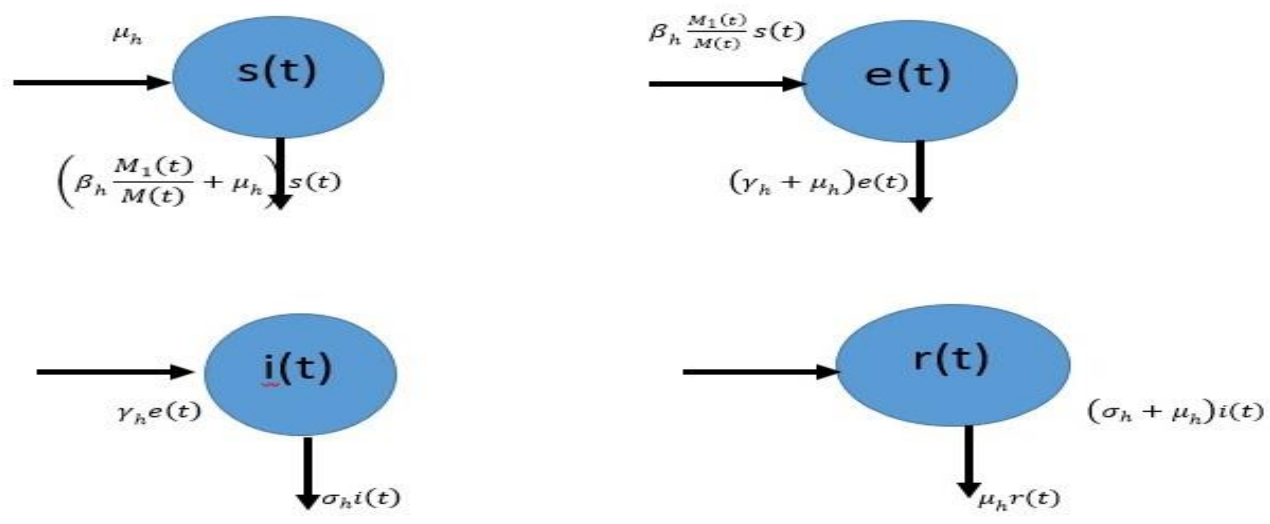

Fig.1 SEIT Dengue Disease Model

The Scheme of Nonlinear Differential Equations(DE) on behalf of the Typical remains specified by:

$$
\begin{aligned}
s^{\prime}(t) & =\mu_{h}-\left(\beta_{h} \frac{M_{1}(t)}{M(t)}+\mu_{h}\right) s(t) \\
e^{\prime}(t) & =\beta_{h} \frac{M_{1}(t)}{M(t)} s(t)-\left(\gamma_{h}+\mu_{h}\right) e(t) \\
r^{\prime}(t) & =\sigma_{h} i(t)-\mu_{h} r(t)
\end{aligned}
$$$$
i^{\prime}(t)=\gamma_{h} e(t)-\left(\sigma_{h}+\mu_{h}\right) i(t)
$$

\section{B: Analysis of the Model}

We describe two equilibrium points of system i.e Disease free equilibrium(DFE) and Endemic equilibrium(EE).

$\varepsilon_{1}=\left(\beta_{h} \frac{M_{1}(t)}{M(t)}, 0,0,0\right)$ and $\quad \varepsilon_{2}=\left(s^{*}, e^{*}, i^{*}, r^{*}\right)$ are stability facts of scheme (1), where

$$
\begin{aligned}
s^{*} & =\frac{\mu_{h}}{\beta_{h} \frac{M_{1}(t)}{M(t)}+\mu_{h}} \\
e^{*} & =\left(\beta_{h} \frac{M_{1}(t)}{M(t)}\right)\left(\frac{\mu_{h}}{\beta_{h} \frac{M_{1}(t)}{M(t)}+\mu_{h}}\right)\left(\frac{1}{\gamma_{h}+\mu_{h}}\right) \\
i^{*} & =\left(\beta_{h} \frac{M_{1}(t)}{M(t)}\right)\left(\frac{\mu_{h}}{\beta_{h} \frac{M_{1}(t)}{M(t)}+\mu_{h}}\right)\left(\frac{\gamma_{h}}{\gamma_{h}+\mu_{h}}\right)\left(\frac{1}{\sigma_{h}+\mu_{h}}\right) \\
r^{*} & =\left(\beta_{h} \frac{M_{1}(t)}{M(t)}\right)\left(\frac{1}{\beta_{h} \frac{M_{1}(t)}{M(t)}+\mu_{h}}\right)\left(\frac{\gamma_{h}}{\gamma_{h}+\mu_{h}}\right)\left(\frac{\sigma_{h}}{\sigma_{h}+\mu_{h}}\right)
\end{aligned}
$$

Where $R_{0}=\frac{\gamma_{h} e(t)}{\sigma_{h}+\mu_{h}}$

$R_{0}$ recognized as Procreative integer who describes the usual number of inferior impurities introduced of the main impurity. $\mathcal{R}_{0}$ is a beginning influence who describe the disease of the exit or persist? If $\mathcal{R}_{0}<1$ then we say that the scheme will observed disease Free Equilibrium (DFE) and iff $\mathcal{R}_{0}>1$ the scheme to involvement Endemic Equilibrium (EE). 


\subsubsection{Numerical Modeling}

Now we have conferred two standard finite difference structures to unravel the endless dynamical scheme (1) i.e. Euler's Method and Runge-Kutta Method of Order 4.

\section{A: Euler Method}

The Forward Euler's Structure for the unceasing model (1) certain through:

$s^{n+1}(t)=s^{n}(t)+h\left[\mu_{h}-\left(\beta_{h} \frac{M_{1}(t)}{M(t)}+\mu_{h}\right) s^{n}(t)\right]$

$e^{n+1}(t)=e^{n}(t)+h\left[\beta_{h} \frac{M_{1}(t)}{M(t)} s^{n}(t)-\left(\gamma_{h}+\mu_{h}\right) e^{n}(t)\right]$

$i^{n+1}(t)=i^{n}(t)+h\left[\gamma_{h} e^{n}(t)-\left(\sigma_{h}+\mu_{h}\right) i^{n}(t)\right]$

$r^{n+1}(t)=r^{n}(t)+h\left[\sigma_{h} i^{n}(t)-\mu_{h} r^{n}(t)\right]$

\section{B:Numerical Experiments}

Now solve numerical tryouts by expending the values of given parameters Table 1 [6].

Table 1

\begin{tabular}{|c|l|l|}
\hline Parameters & Values \\
\cline { 2 - 3 } & DFE & EE \\
\hline$\mu_{h}$ & 0.000042 & 0.00042 \\
\hline$\beta_{h}$ & 0.375 & 0.750 \\
\hline$\gamma_{h}$ & 0.100 & 0.200 \\
\hline$\sigma_{h}$ & 0.143 & 0.140 \\
\hline$M_{1}(t)$ & 1 & 1 \\
\hline$M(t)$ & 0.330 & 0.330 \\
\hline
\end{tabular}

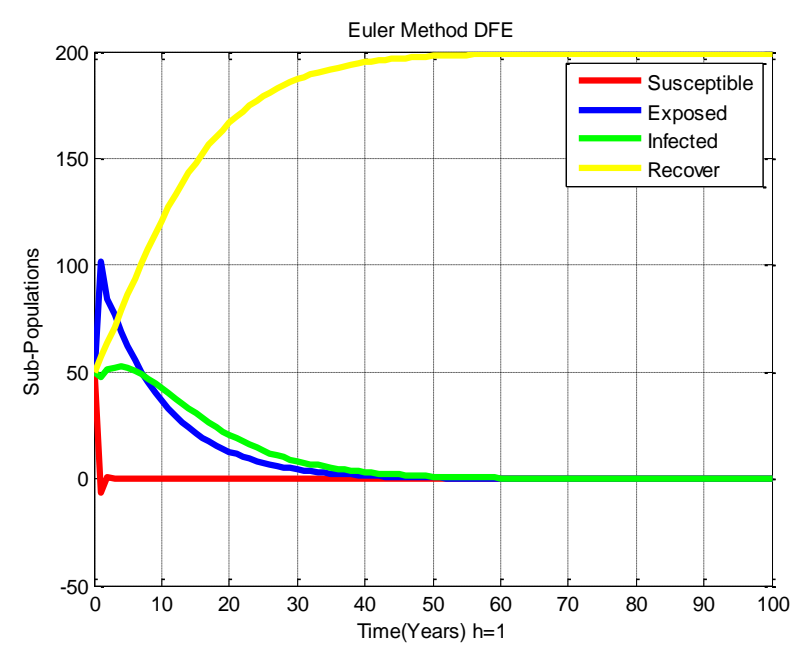

Fig. 2 Euler Method (DFE), $h=1$ 


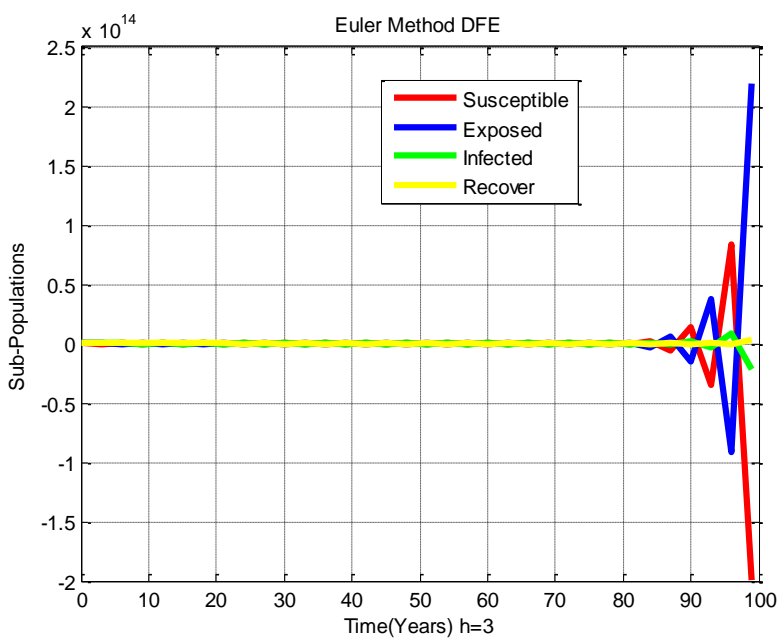

Fig.3 Euler Method (DFE), $h=3$

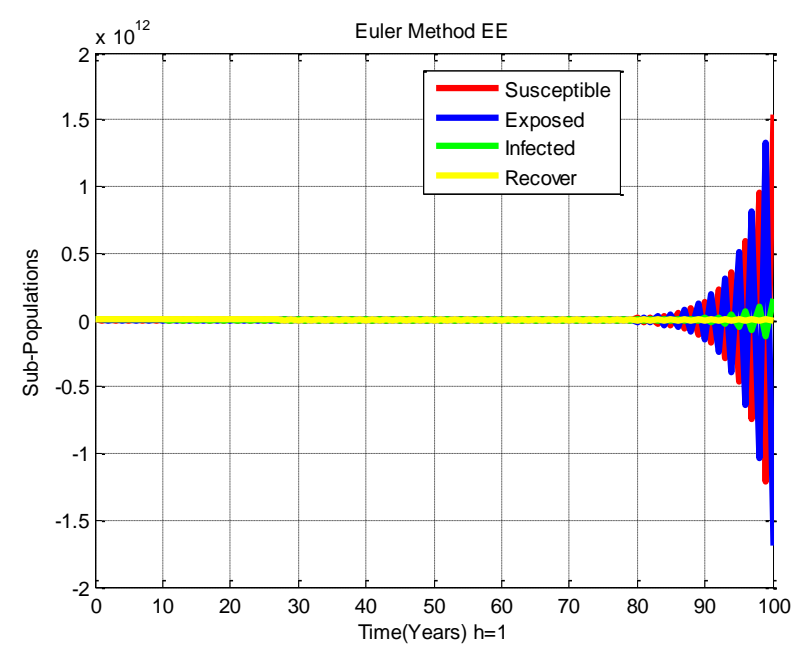

Fig. 4 Euler Method (EE), $h=1$ 


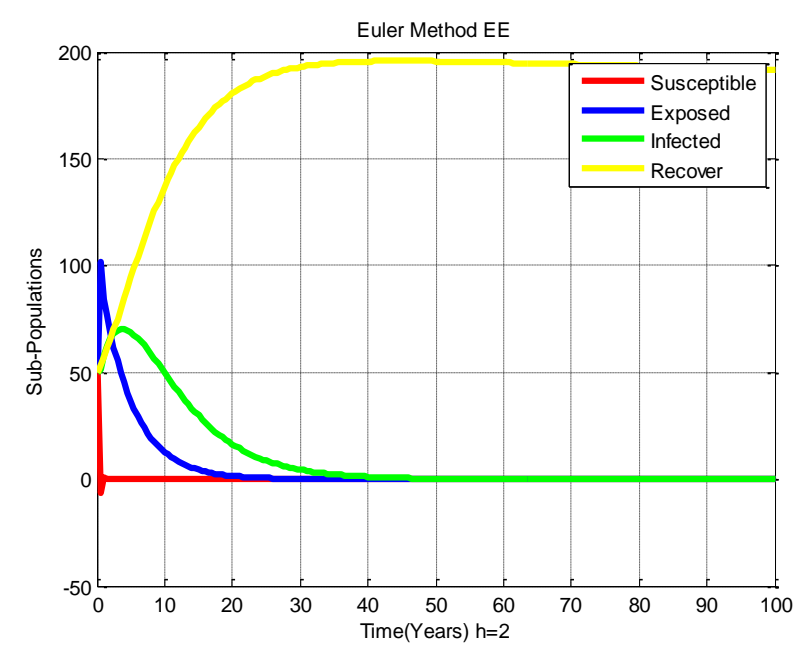

Fig.5 Euler Method (EE), $h=0.5$

\section{C: $\quad$ Fourth Order Runge-Kutta Scheme}

For Stage-1

$k_{1}=h\left[\mu_{h}-\left(\beta_{h} \frac{M_{1}(t)}{M(t)}+\mu_{h}\right) s^{n}(t)\right]$

$l_{1}=h\left[\beta_{h} \frac{M_{1}(t)}{M(t)} s^{n}(t)-\left(\gamma_{h}+\mu_{h}\right) e^{n}(t)\right]$

$m_{1}=h\left[\gamma_{h} e^{n}(t)-\left(\sigma_{h}+\mu_{h}\right) i^{n}(t)\right]$

$n_{1}=h\left[\sigma_{h} i^{n}(t)-\mu_{h} r^{n}(t)\right]$

For Stage-2

$k_{2}=h\left[\mu_{h}-\left(\beta_{h} \frac{M_{1}(t)}{M(t)}+\mu_{h}\right)\left(s^{n}(t)+\frac{k_{1}}{2}\right)\right]$

$l_{2}=h\left[\beta_{h} \frac{M_{1}(t)}{M(t)}\left(s^{n}(t)+\frac{k_{1}}{2}\right)-\left(\gamma_{h}+\mu_{h}\right)\left(e^{n}(t)+\frac{l_{1}}{2}\right)\right]$

$m_{2}=h\left[\gamma_{h}\left(e^{n}(t)+\frac{l_{1}}{2}\right)-\left(\sigma_{h}+\mu_{h}\right)\left(i^{n}(t)+\frac{m_{1}}{2}\right)\right]$

$n_{2}=h\left[\sigma_{h}\left(i^{n}(t)+\frac{m_{1}}{2}\right)-\mu_{h}\left(r^{n}(t)+\frac{n_{1}}{2}\right)\right]$

For Stage-3

$k_{3}=h\left[\mu_{h}-\left(\beta_{h} \frac{M_{1}(t)}{M(t)}+\mu_{h}\right)\left(s^{n}(t)+\frac{k_{2}}{2}\right)\right]$

$l_{3}=h\left[\beta_{h} \frac{M_{1}(t)}{M(t)}\left(s^{n}(t)+\frac{k_{2}}{2}\right)-\left(\gamma_{h}+\mu_{h}\right)\left(e^{n}(t)+\frac{l_{2}}{2}\right)\right]$ 


$$
\begin{aligned}
& m_{3}=h\left[\gamma_{h}\left(e^{n}(t)+\frac{l_{2}}{2}\right)-\left(\sigma_{h}+\mu_{h}\right)\left(i^{n}(t)+\frac{m_{2}}{2}\right)\right] \\
& n_{3}=h\left[\sigma_{h}\left(i^{n}(t)+\frac{m_{2}}{2}\right)-\mu_{h}\left(r^{n}(t)+\frac{n_{2}}{2}\right)\right]
\end{aligned}
$$

\section{For Stage-4}

$$
\begin{aligned}
& k_{4}=h\left[\mu_{h}-\left(\beta_{h} \frac{M_{1}(t)}{M(t)}+\mu_{h}\right)\left(s^{n}(t)+k_{3}\right)\right] \\
& l_{4}=h\left[\beta_{h} \frac{M_{1}(t)}{M(t)}\left(s^{n}(t)+k_{3}\right)-\left(\gamma_{h}+\mu_{h}\right)\left(e^{n}(t)+l_{3}\right)\right] \\
& m_{4}=h\left[\gamma_{h}\left(e^{n}(t)+l_{3}\right)-\left(\sigma_{h}+\mu_{h}\right)\left(i^{n}(t)+m_{3}\right)\right] \\
& n_{3}=h\left[\sigma_{h}\left(i^{n}(t)+m_{3}\right)-\mu_{h}\left(r^{n}(t)+n_{3}\right)\right]
\end{aligned}
$$

Finally

$$
\begin{aligned}
& s^{n+1}=s^{n}+\frac{1}{6}\left[k_{1}+2 k_{2}+2 k_{3}+k_{4}\right] \\
& e^{n+1}=e^{n}+\frac{1}{6}\left[l_{1}+2 l_{2}+2 l_{3}+l_{4}\right] \\
& i^{n+1}=i^{n}+\frac{1}{6}\left[m_{1}+2 m_{2}+2 m_{3}+m_{4}\right] \\
& r^{n+1}=r^{n}+\frac{1}{6}\left[n_{1}+2 n_{2}+2 n_{3}+n_{4}\right]
\end{aligned}
$$

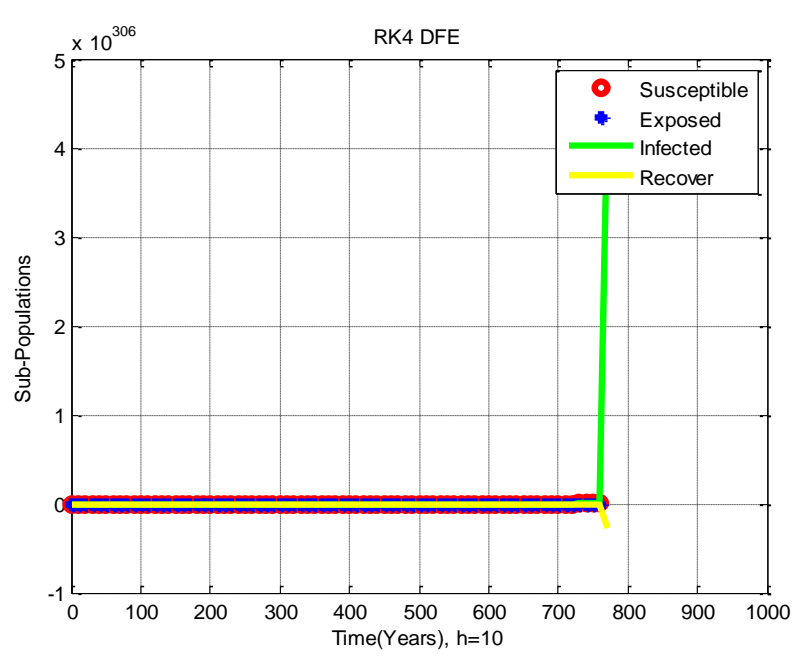

Fig.6 RK-4 Method (DFE), $h=10$ 


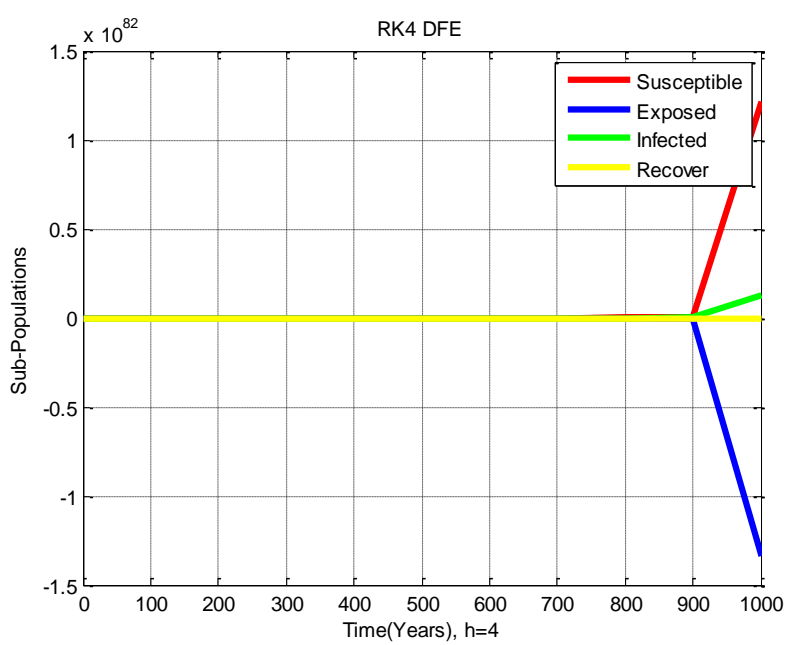

Fig.7 RK-4 Method (DFE), $h=4$

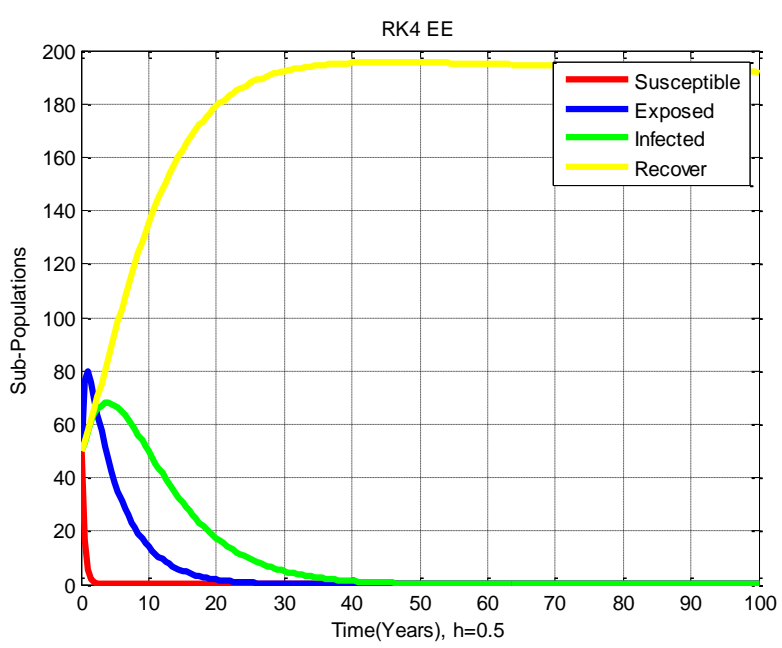

Fig.8 RK-4 Method (EE), $h=200$

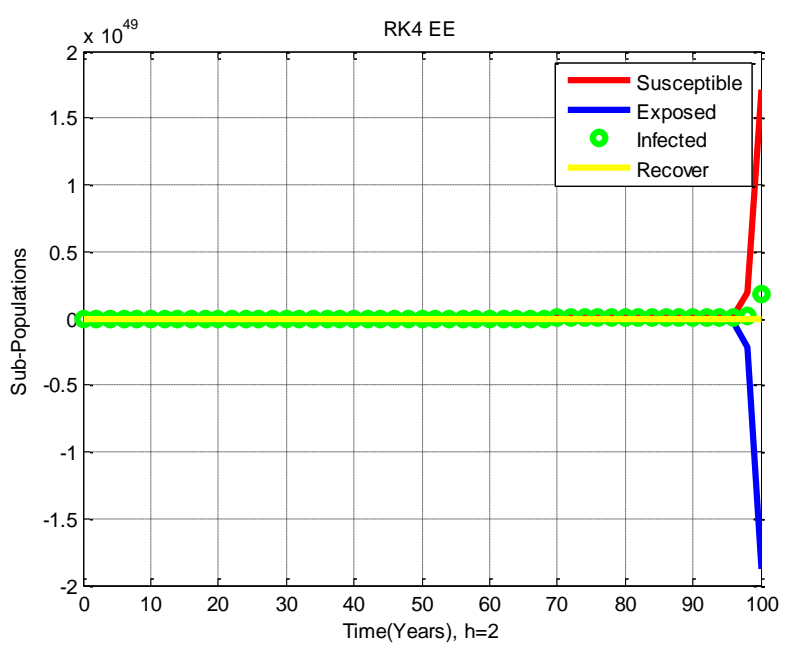

Fig.9 RK-4 Method (EE), $h=2$ 


\section{C.Non-standard Finite DIFFERENCE MODEL}

We display absolutely convergent non-standard finite difference(NSFD). So that the covergenence learning of the recommended building. So

$$
\begin{aligned}
s^{n+1} & =\frac{s^{n}(t)+h \mu_{h}}{1+h\left(\beta_{h} \frac{M_{1}(t)}{M(t)}+\mu_{h}\right)} \\
e^{n+1} & =\frac{e^{n}(t)+h \beta_{h} \frac{M_{1}(t)}{M(t)} s^{n}(t)}{1+h\left(\gamma_{h}+\mu_{h}\right)} \\
i^{n+1} & =\frac{i^{n}(t)+h \gamma_{h} e^{n}(t)}{1+h\left(\sigma_{h}+\mu_{h}\right)} \\
r^{n+1} & =\frac{r^{n}(t)+h \sigma_{h} i^{n}(t)}{1+h \mu_{h}}
\end{aligned}
$$

A: Convergence Analysis of NSFD Scheme

Let us define

$E=\frac{s+h \mu_{h}}{1+h\left(\beta_{h} \frac{M_{1}(t)}{M(t)}+\mu_{h}\right)}$

$F=\frac{e+h \beta_{h} \frac{M_{1}(t)}{M(t)} s}{1+h\left(\gamma_{h}+\mu_{h}\right)}$

$G=\frac{i+h \gamma_{h} e}{1+h\left(\sigma_{h}+\mu_{h}\right)}$

$$
H=\frac{r+h \sigma_{h} i}{1+h \mu_{h}}
$$

Now the Jacobian Matrix is given by

$$
J=\left[\begin{array}{llll}
\frac{\partial E}{\partial s} & \frac{\partial E}{\partial e} & \frac{\partial E}{\partial i} & \frac{\partial E}{\partial r} \\
\frac{\partial F}{\partial s} & \frac{\partial F}{\partial e} & \frac{\partial F}{\partial i} & \frac{\partial F}{\partial r} \\
\frac{\partial G}{\partial s} & \frac{\partial G}{\partial e} & \frac{\partial G}{\partial i} & \frac{\partial G}{\partial r} \\
\frac{\partial H}{\partial s} & \frac{\partial H}{\partial e} & \frac{\partial H}{\partial i} & \frac{\partial H}{\partial r}
\end{array}\right]
$$

At DiseaseFree Equilibrium $\mathcal{E}_{1}=\left(\beta_{h} \frac{M_{1}(t)}{M(t)}, 0,0,0\right)$

At Endemic Equilibrium

$$
\begin{aligned}
& \mathcal{E}_{1}=\left(\frac{\mu_{h}}{\beta_{h} \frac{M_{1}(t)}{M(t)}+\mu_{h}},\left(\beta_{h} \frac{M_{1}(t)}{M(t)}\right)\left(\frac{\mu_{h}}{\beta_{h} \frac{M_{1}(t)}{M(t)}+\mu_{h}}\right)\left(\frac{1}{\gamma_{h}+\mu_{h}}\right),\left(\beta_{h} \frac{M_{1}(t)}{M(t)}\right)\left(\frac{\mu_{h}}{\beta_{h} \frac{M_{1}(t)}{M(t)}+\mu_{h}}\right)\left(\frac{\gamma_{h}}{\gamma_{h}+\mu_{h}}\right)\left(\frac{1}{\sigma_{h}+\mu_{h}}\right),\right. \\
& \left.\left(\beta_{h} \frac{M_{1}(t)}{M(t)}\right)\left(\frac{1}{\beta_{h} \frac{M_{1}(t)}{M(t)}+\mu_{h}}\right)\left(\frac{\gamma_{h}}{\gamma_{h}+\mu_{h}}\right)\left(\frac{\sigma_{h}}{\sigma_{h}+\mu_{h}}\right)\right)
\end{aligned}
$$




\section{B: Numerical Experiments}

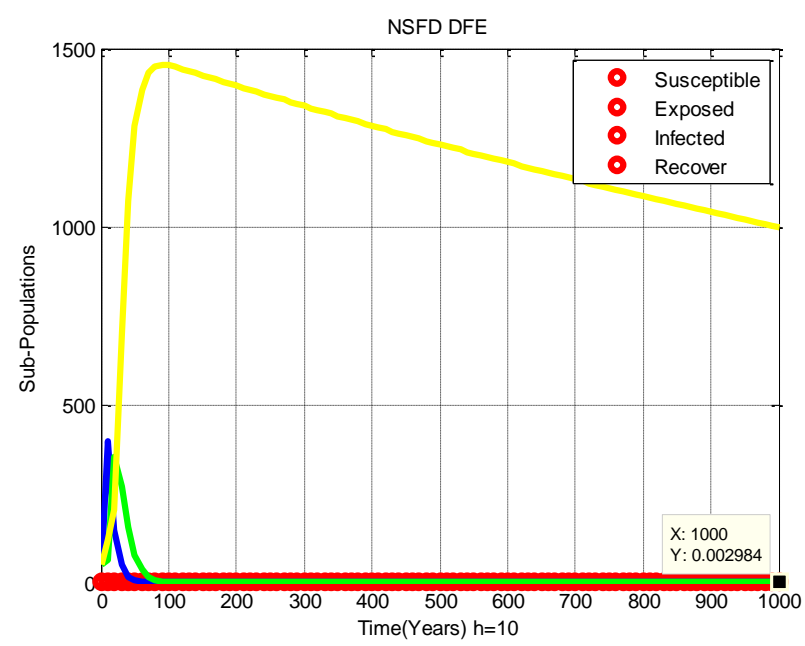

Fig.10 NSFD Method (DFE), $h=10$

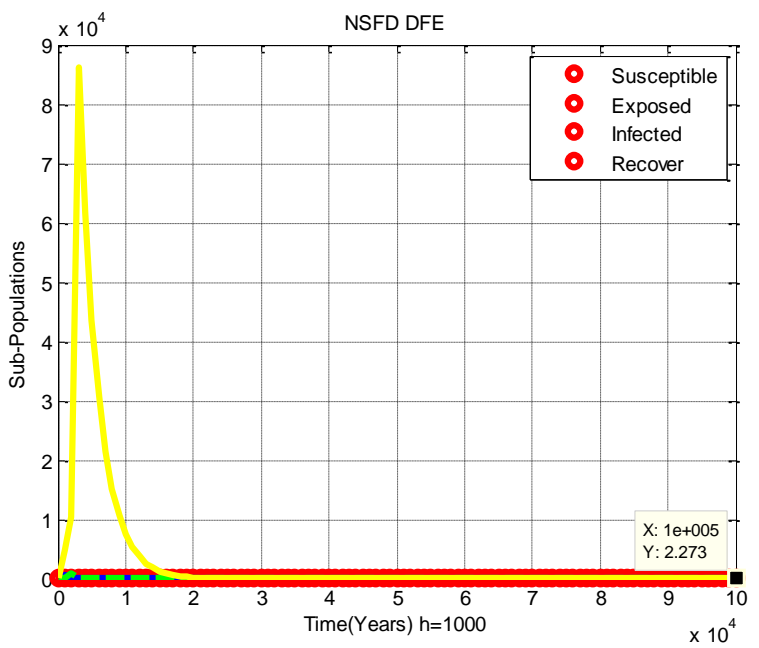

Fig.11 NSFD Method (DFE), $h=1000$ 


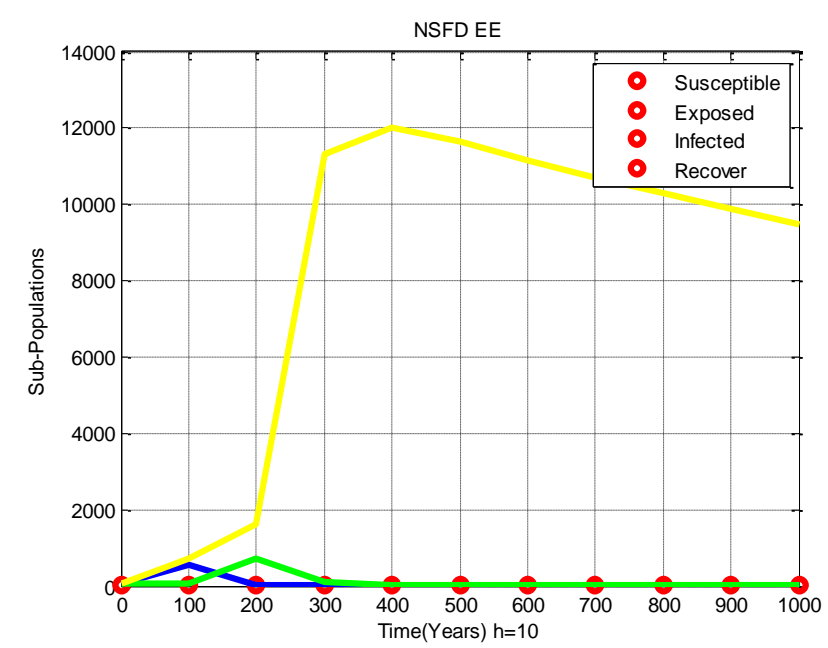

Fig.12 NSFD Method (EE), $h=10$

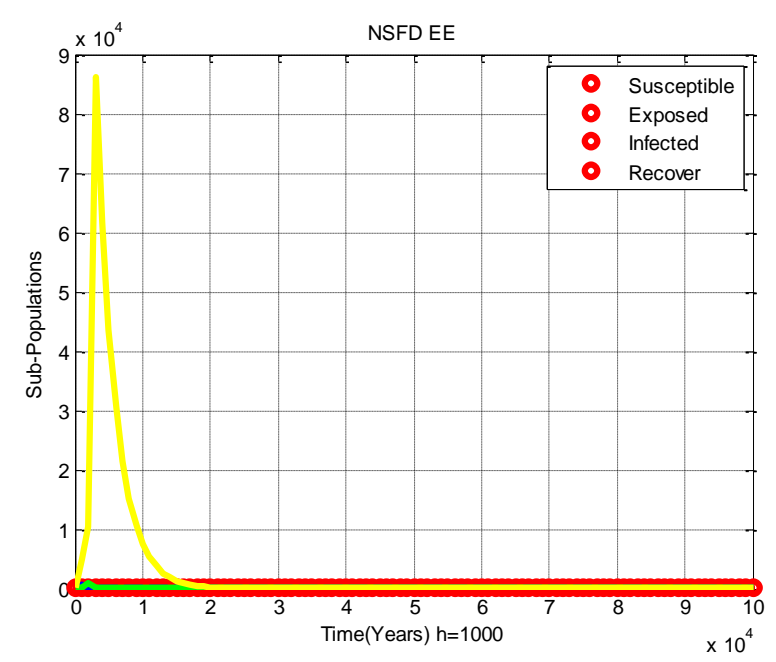

Fig.13 NSFD Method (EE), $h=1000$

\section{D: Comparison Analysis}

In this section, we see the comparison among of two standard difference schemes and non-standard difference scheme in epidemiology 


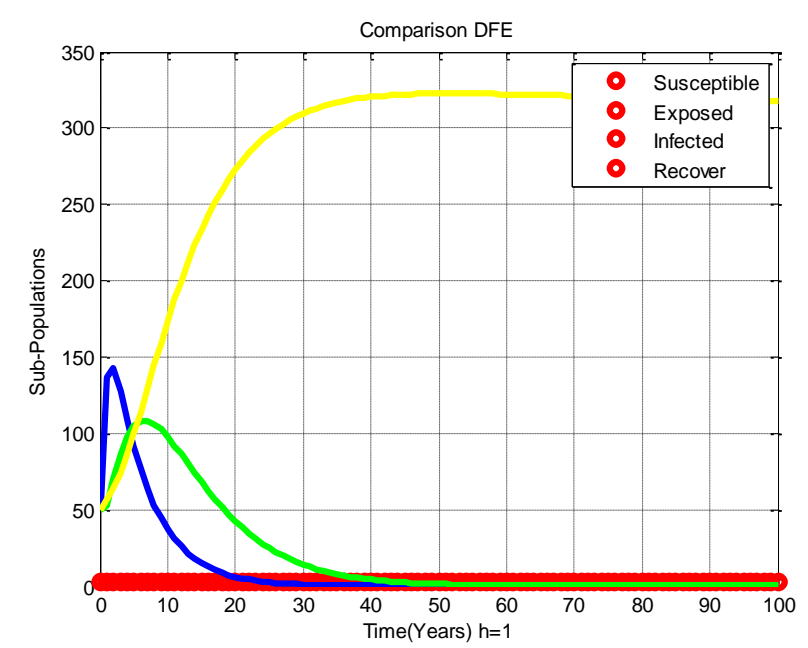

Fig.14 comparison (DFE), $h=1$

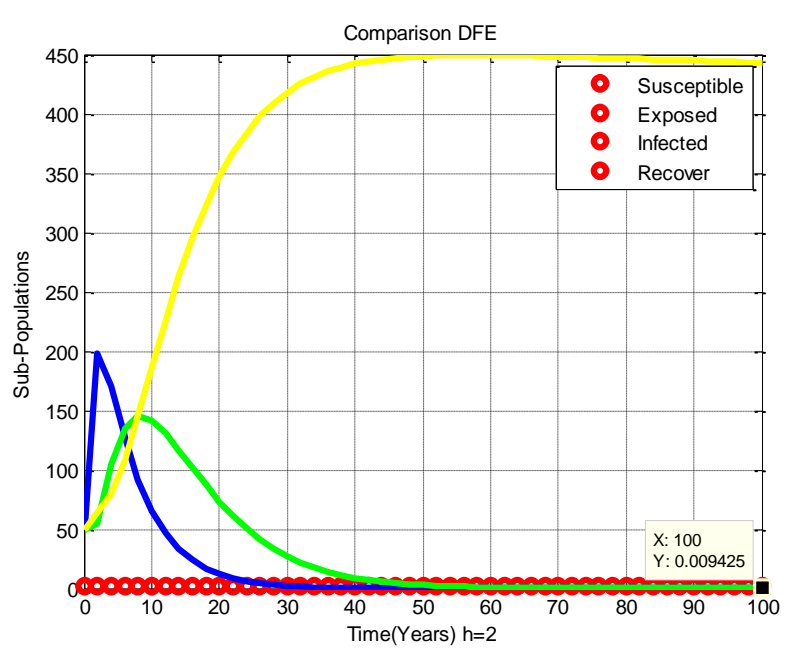

Fig.15 comparison (DFE), $h=2$

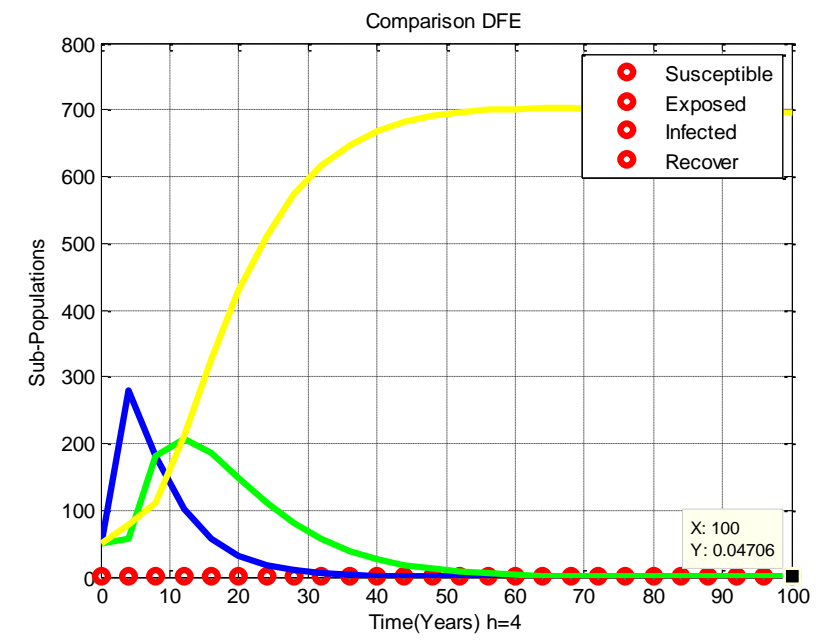

Fig.16 comparison (DFE), $h=4$ 


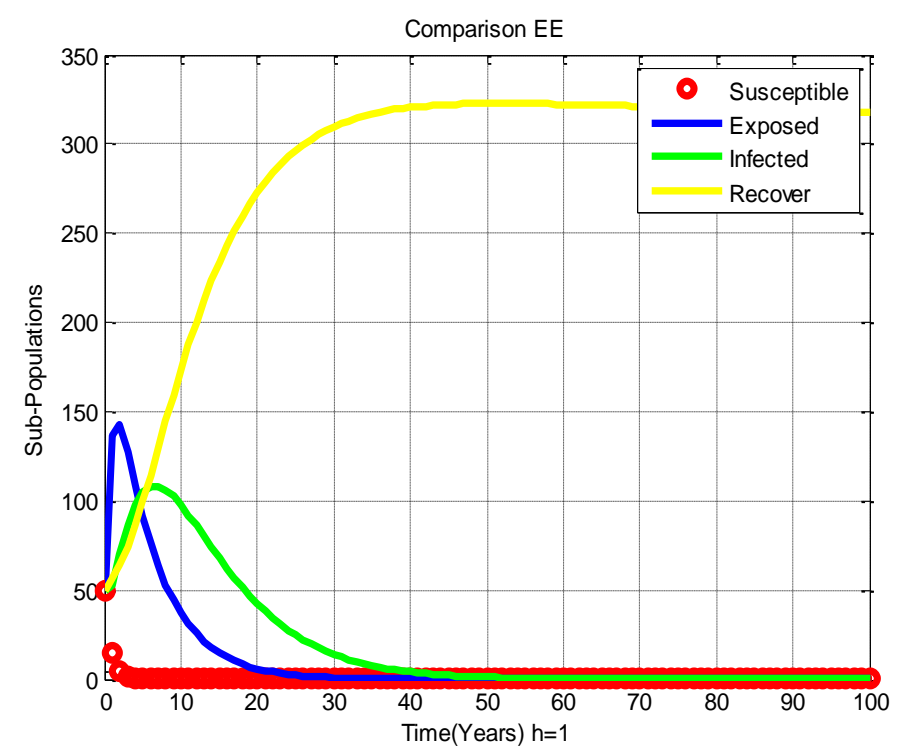

Fig.17 comparison (EE), $h=1$

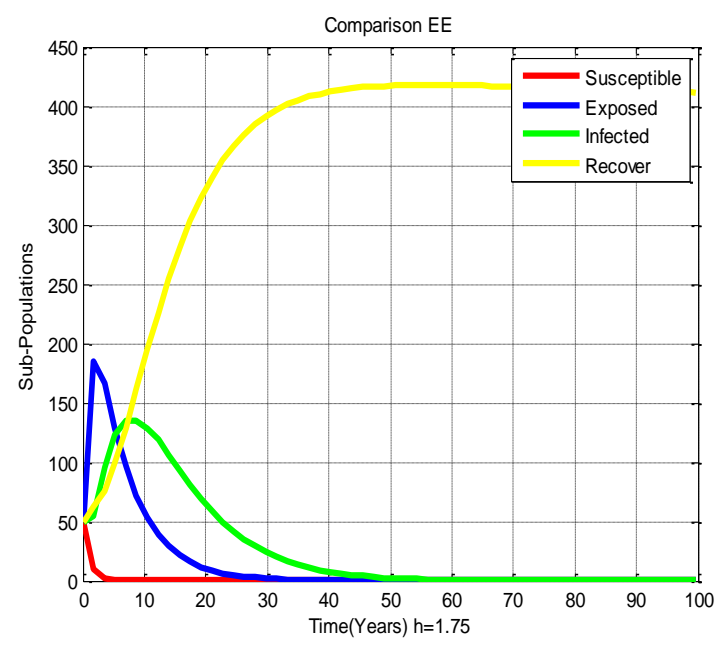

Fig.18 comparison $(\mathrm{EE}), \mathrm{h}=\mathbf{1 . 7 5}$ 


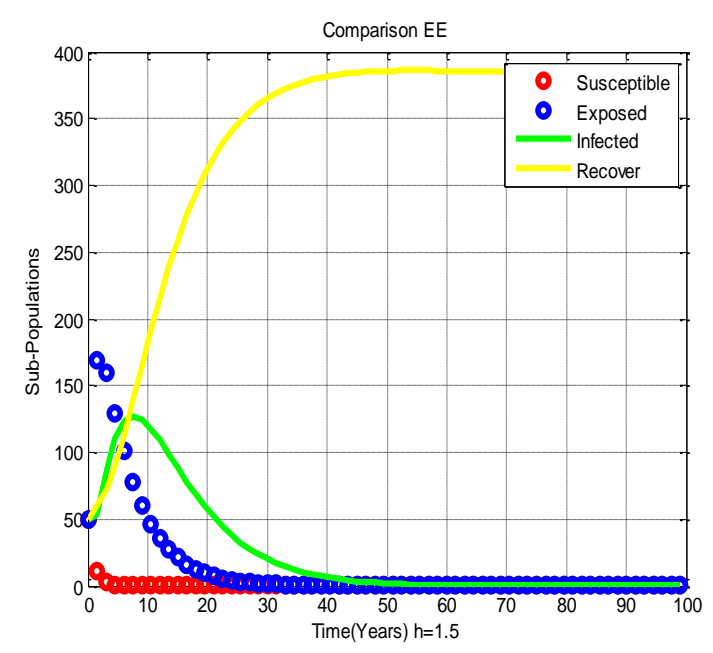

Fig.19 comparison (EE), $\mathrm{h}=1.5$

\subsubsection{Results and Discussion}

The model of dengue consumes introduced expending SEIT Model. (i.e Susceptible, Exposed, Infected and Treated). The loyalty of firm spots i.e the Disease free equilibrium(DFE) and Endemic equilibrium truths(EE) thought arithmetically. So label an unqualifiedly endless Non-Standard Finite Difference (NSFD) arrangement the continual dynamical scheme.The optional construction happens dynamical consistant, arithmetically secure and grips athentic possessions of the relentless model. The outcomes equaled well known standard finite difference schemes i.e Euler's and Runge-Kutta method of order 4 (RK-4). The Euler and RK-4 be influenced by step size ' $h$ ' while the constructed NSFD scheme for every assessment used to scums convergent.

\subsubsection{Conclusion}

The Non-Standard Finite Difference Scheme shaped aimed at dengue Euler and RK-4 are failed because they be depend value h. So Euler and RK-4 are temporarily convergent. Euler and RK-4 are divergent and change answer via value of $\mathrm{h}$. But Non Standard Finite Difference Scheme is independent on value h. Improbability the step size in hundreds and thousands then NSFD still convergent. NSFD Scehme satisfy all convergent properties. The graphical behaviour Euler, RK-4 and NSFD schemes are in figure no.1 to 19. The compassion of differences the condensed amount of than the other assemblies. So sign that NSFD is unswerving.

\section{References}

1. E. B.Wilson, J.Woecester, The law of mass action in epidemiology, Proc. Natl. Acad. Sci., USA, 31 (1945) 24-34 (part I) 109-116 (part II).

2. G. Macdonald, The Epidemiology and Control of Malaria, London: Oxford University Press, 1957.

3. L. Esteva, C. Vargas, Analysis of a dengue disease transmission model, Math. Biosci., 150 (1998) 131 151.

4. A. J. johnson, J. T. Roehring, New mouse model for dengue virus vaccine testing, The Jour. of Virol., 73 (1) (1999) 783-786. 
5. I. kurane, T. Takasaki, Dengue fever and dengue hemorrhagic fever: challenges of contolling an enemy still at large, Rev. Med. Virol., 11 (5) (2001) 301-311.

6. D. J. Gubler, The global emergence/resurgence of arboviral disease as public health problems, Arc. Med. 33 (4) (2002), 330-342.

7. M. Ghosh, p. Chandra, P. Sinha, J. B. Shukla, Modelling the spread of bacterial disease: e_ect of service providers from an environmentally degraded region, Appl. Math. Comput. 160 (2005) 615647.

8. S. Singh, J. B. Shukla, P. Chandra, Modelling and analysis of the spread of malaria: Environmental and ecological e_ects, J. Bio. Syst. 13(1) (2005) 111.

9. H. M. Yang, C. P. Ferreira, Assessing the e_ects of the vector control on dengue transmission, Appl. Math. Comput., 198 (2008) 401-413.

10. M. N. Burattini, M. Chen, A. Chow, F. A. Coutinho, K. T. Goh, L. F. Lopez, S. Ma, E. Massad, Modelling the control strategies against dengue in Singapure, Epidemiol Infect., 136 (3) (2008) 309 319.

11. P. M. Luz, C. T. Codec,o, J. Medlock, C. J. Struchiner, D. Valle, A. P. Galvani, Profile of insecticide interventions on the abundance and resistence profile ofAedes aegypti, Epidemiol. Infect., 137 (8) (2009) 1203-1215.

12. N. A. Maidana, H. M. Yang, Spatial spreading of West Nile Virus described by traveling waves, Jour. of Theor. Biol., 258 (2009) 403417.

13. A. Korobeinicov, Global properties of SIR and SEIR epidemic models with multiple parallel interactions stages, Bull. Math. Bio., 71 (2009) 75-83.

14. J. G. Julander, S. T. Perry, S. Shresta, Important advances in the field of anti-dengue virus research, Antivir. Chem. Chemother, 21 (3) (2011) 105-116.

15. M. A. Johansson, J. Hombach, D. A. Cummings, Models of the impact of dengue vaccines: a review of current research and potential approaches, Vaccine, 29 (35) (2011) 5860-5868.

16. M. B. Arduino, Assessment of Aedes aegypti pupal productivity during the dengue vector control program in a costal urban centre of S£o Paulo state, Brazil, Jour. of Insec., 2014 (2014) 1-9. 